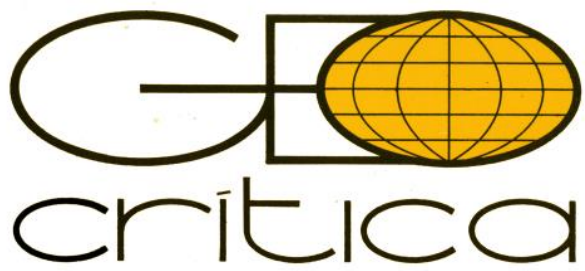

240, marzo de 2020

\title{
ESTUDOS E PESQUISAS SOBRE O USO DE PLANTAS MEDICINAIS E FITOTERÁPICOS NO BRASIL: CAMINHOS E DESAFIOS
}

\author{
Marta Rocha de Castro \\ Departamento de Geografia, Pontifícia Universidade Católica do Rio de Janeiro, Brasil \\ martarochaacupuntura@gmail.com \\ Fábio Fonseca Figueiredo \\ Departamento de Políticas Públicas, Universidade Federal do Rio Grande do Norte, Brasil \\ ffabiof@yahoo.com
}

\section{Estudos e pesquisas sobre o uso de plantas medicinais e fitoterápicas no Brasil: caminhos e desafios (Resumo)}

A medicina tradicional e popular é uma prática secular realizada pelos povos autóctones e outras populações tradicionais. Esse saber popular foi repassado oralmente de uma geração a outra. No final da década de 1970, as organizações internacionais de saúde passaram a se interessar por essa forma de cuidado e atenção e, então, ocorreu a institucionalização das medicinas alternativas e complementares e, dentre elas, o uso de plantas medicinais e fitoterápicos. Com o objetivo de contribuir com o debate contemporâneo sobre o tema, o texto traz uma reflexão sobre a trajetória do uso de plantas medicinais e fitoterápicos como forma de cuidado com a saúde no Brasil. O presente artigo apresenta algumas páginas eletrônicas brasileiras sobre o tema bem como recomenda alguns portais hospedados fora do país.

Palavras chave: Fitoterápicos, Geografia da saúde, Medicina tradicional, Plantas medicinais, Sistema Único de Saúde.

\section{Studies and research on the use of medicinal and herbal plants in Brazil: ways and challenges (Abstract)}

Traditional and popular medicine is a secular practice performed by indigenous peoples and other traditional populations and that popular knowledge is passed on through generations through oral history and diverse records. In the late 1970s, international public health organizations became interested in this form of health care and care, and then the institutionalization of alternative and complementary medicines, 
including the use medicinal plants and herbal medicines. Waiting to contribute to the contemporary debate about the theme, the text shows a reflection on the trajectory of the use of medicinal plants and herbal medicines as a form of health care in Brazil. Therefore, this article presents some web pages produced in the country on the subject as well as recommends some portals hosted outside the country.

Key words: Phytotherapeutics, Health geography, Traditional medicine, Medicinal plants, Health Unic System.

\section{Estudios e investigaciones sobre el uso de plantas medicinales y herbales en Brasil: caminos y desafíos (Resumen)}

La medicina tradicional y popular es una práctica secular realizada por los pueblos autóctonos y otras poblaciones tradicionales y ese saber popular es repasado entre generaciones a través de la historia oral y registros diversos. A finales de la década de 1970, las organizaciones internacionales de salud pública pasaron a interesarse por esa forma de cuidado y atención a la salud y entonces ocurrió la institucionalización de las medicinas alternativas y complementarias, y entre ellas el uso de plantas medicinales y fitoterápia. Para contribuir al debate contemporáneo sobre el tema, el texto muestra una reflexión sobre la trayectoria del uso de plantas medicinales y hierbas medicinales como una forma de atención de la salud en Brasil. Por lo tanto, él artículo presenta algunas páginas web producidas en el país sobre el tema y recomienda algunos portales hospedados fuera del país.

Palabras clave: Fitoterápia, Geografía de la salud, Medicina tradicional, Plantas medicinales, Sistema Único de Salud.

No final da década de 1980 estimava-se que $80 \%$ dos habitantes de países em desenvolvimento dependiam da medicina popular para suprir suas necessidades básicas de saúde, e que $85 \%$ dos medicamentos produzidos pela medicina tradicional e popular envolviam o uso de extratos de plantas e ervas, portanto remédios produzidos com produtos da natureza próxima de onde residem esses habitantes ${ }^{1}$. Estes números mostram a relevância das práticas integrativas a partir das culturas tradicionais bem como a importância do uso das plantas medicinais e fitoterápicos como forma de cuidado e atenção à saúde básica. Mostram também a eficácia desse tipo de tratamento médico bem como a importância da biodiversidade local para a saúde humana através da utilização dos métodos científicos que comprovam a eficácia das plantas medicinais e fitoterápicos reforçando o seu uso nos cuidados com a saúde ${ }^{2}$.

Foi a partir dos anos 1970 que organismos internacionais tais como a Organização Mundial de Saúde (OMS) e a Organização Pan-Americana de Saúde (OPAS) passaram a se interessar pelo uso de plantas medicinais nos cuidados com a saúde. Neste mesmo período, nos países mais desenvolvidos, observamos o aumento no consumo de fitoterápicos e crescimento dos mercados verdes (green grabbing), influenciados pelos movimentos sociais da época, que contestavam o modelo biomédico e a valorização da ciência e da técnica em detrimento da desvalorização do mundo natural ${ }^{3}$.

1 Alho, $2012<$ http://www.scielo.br/pdf/ea/v26n74/a11v26n74.pdf〉.

2 Castro e Figueiredo, $2019<$ http://www.seer.ufu.br/index.php/hygeia/article/view/46605>.

3Nascimento et. al., 2013 <https://www.scielosp.org/article/csc/2013.v18n12/3595-3604/>. 
Estudos e pesquisas sobre o uso de plantas medicinais e fitoterápicos...

No Brasil, o uso das plantas medicinais como recomendação de política pública se sistematizou a partir de 2006 com a institucionalização da Política Nacional de Plantas Medicinais e Fitoterápicos pelo Ministério da Saúde.

A relevância do uso de plantas medicinais e fitoterápicos no cuidado à saúde básica conduz às pesquisas no sentido de, inicialmente, dar maior visibilidade ao próprio tema, ainda emudecido por estar preterido por parte dos profissionais da área da saúde e uma parcela da população. Entendemos que o tema deve ser tratado com o devido respeito e atenção haja vista que, ao menos no senso comum e coadunado por uma estratégia nefasta de menosprezo, a difusão de saberes e práticas tradicionais, associadas às práticas alternativas de saúde tais como o uso de plantas medicinais são encaradas como crendice e até charlatanismo por parte de quem defende essa forma de cura.

Assim, precisam ser revistos e reavaliados preceitos tais como a descrença no uso das plantas medicinais no tratamento a enfermos e as dúvidas quanto às práticas oriundas do saber tradicional. Paralelamente, as instituições públicas e privadas devem fomentar as pesquisas que comprovem a eficácia das ervas com a finalidade de fortalecer a indústria de fitoterápicos, um dos principais objetivos da Política de Plantas Medicinais e Fitoterápicos.

Este artigo, que se insere no âmbito da geografia da saúde, pretende contribuir com a difusão do conhecimento sobre o uso de plantas medicinais e fitoterápicos através de indicações de referências que podem ser úteis para pesquisadores da área e demais profissionais que se interessem pela temática, além de pessoas interessadas na busca de fontes fidedignas sobre o tema. Para tanto, faz-se primordial a formação de uma literatura acadêmica que trate do uso de plantas medicinais e fitoterápicos despida dos modismos impregnados nos diversos trabalhos já produzidos e que reduzem essa forma de tratamento como crendice popular, quando não a tratam como uma forma de charlatanismo da parte dos seus defensores e praticantes.

A particularidade deste artigo está na perspectiva da interdisciplinaridade, e, nesse sentido, é factível a contribuição da ciência geográfica na consolidação do estudo, já que "para el entendimiento de los fenómenos físicos y sociales se requiere la interacción entre las ciencias sociales y humanas".

As páginas eletrônicas que apresentaremos são de fácil e praticamente acesso irrestrito, com mérito reconhecido por pesquisadores que investigam o tema justamente por disponibilizar informações confiáveis, de tal modo que instiga o pesquisador e o interessado pelo tema a refletir sobre as questões que envolvem o uso de planta medicinais na contemporaneidade.

Além desta introdução, o texto está dividido em outras duas secções. A primeira apresenta e analisa a institucionalização do uso de plantas medicinais e fitoterápicos pelos países membros da OMS, a partir das recomendações da própria Organização Mundial da Saúde e da Organização Pan-Americana de Saúde. Analisa, igualmente, a polarização na forma de tratamento médico, colocando de um lado a medicina convencional baseada na medicalização, tecnologização e a simplificação biológica da doença; e de outro, a medicina tradicional e popular, que parte de uma visão integrativa e subjetiva do processo de adoecimento, fundamentando-se em aspectos tais

4 Capel, $1998<$ http://www.ub.es/geocrit/sn-19.htm>. 
como a cura através do uso de plantas medicinais e fitoterápicos e o autocuidado/autoatenção do usuário. Na segunda secção, analisamos diversas Webs que versam sobre o uso de plantas medicinais e fitoterápicos em cinco subdivisões: Documentos governamentais, Portais de pesquisa de instituições públicas, Revistas científicas, Organizações não governamentais e Entidades do terceiro setor e Webs nacionais e internacionais. Finalmente, as considerações finais deixam em aberto o debate sobre o tema exposto.

\section{A institucionalização do uso de plantas medicinais e fitoterápicos nos sistemas públicos de saúde}

O progresso tecnológico da medicina e o desenvolvimento e alcance da indústria farmacêutica global fizeram com que os tratamentos baseados em plantas medicinais e fitoterápicos fossem relegados ao segundo plano nos sistemas de saúde públicos. Esses tratamentos alternativos foram interpretados como uma forma de cuidado à saúde relacionado a crendices populares, portanto, sem validação científica necessária à ciência médica. Na sua pesquisa sobre perspectivas e desafios do uso de plantas medicinais e fitoterápicos no Sistema Único de Saúde (SUS), Climério Avelino de Figueredo e colaboradores ${ }^{5}$ analisam que a indústria farmacêutica teve o seu primeiro pico de desenvolvimento por volta dos anos 1960 e, uma das formas de mostrar a eficiência e eficácia dos seus medicamentos sintéticos foi através da desqualificação do saber popular sobre as plantas medicinais.

Para os autores acima citados, o lobby da indústria farmacêutica contra os fitoterápicos não se fundamentou somente na suposta inferioridade da eficácia na cura à enfermidades e elevada toxidade das plantas medicinais em comparação com os medicamentos sintéticos, e tampouco na falta de comprovação científica dos saberes e práticas populares. O lobby da indústria farmacêutica foi formado devido aos interesses mercadológicos daquela indústria, que se expandia e usava como uma de suas principais estratégias a desvalorização e desqualificação da medicina tradicional bem como o uso de plantas e fitoterápicos.

Por outro lado, foi também durante as décadas de 1960 e 1970 que os diversos movimentos contraculturais em países como Estados Unidos, França e Alemanha reivindicavam novos e distintos padrões de sociabilidade que se diferenciassem da sociedade industrial capitalista que se formara até então. No âmbito da saúde, houve uma busca pelos cuidados médicos através de tratamentos baseados em práticas tradicionais utilizadas há séculos por populações tradicionais. Sob influência destes movimentos foi criado o termo Racionalidades Médicas (RM), que emergiu no campo da saúde coletiva, área das ciências sociais e humanas em saúde. O projeto RM propôs a existência de outras racionalidades médicas não apenas a ocidental/biomedicina e que coexistem na cultura atual, tais como as medicinas tradicionais e a homeopatia ${ }^{6}$.

A institucionalização de tais demandas sociais para o setor da saúde ocorreu no final dos anos 1970, quando a Organização Mundial de Saúde (OMS) e a Organização Pan-Americana de Saúde

\footnotetext{
5 Figueredo et. al., $2014 \quad<$ http://www.scielo.br/scielo.php?script=sci_arttext\&pid=S0103$73312014000200381 \& \operatorname{lng}=\mathrm{en} \& \mathrm{nrm}=$ iso $>$.

6 Nascimento et. al., 2013 <https://www.scielosp.org/article/csc/2013.v18n12/3595-3604/>.
} 
Estudos e pesquisas sobre o uso de plantas medicinais e fitoterápicos...

(OPAS) se pronunciaram sobre o assunto e lançaram o "Programa de Medicina Tradicional". Conforme Marcelo Saad e Paulo de Tarso Lima:

Desde então, em vários comunicados e resoluções, a OMS expressa o seu compromisso em incentivar os Estados-membros a formular e implementar políticas públicas para uso racional e integrado da Medicina tradicional e de terapias complementares nos sistemas nacionais de atenção à saúde básica, bem como para o desenvolvimento de estudos científicos para melhor conhecimento de sua segurança, eficácia e qualidade ${ }^{7}$.

Em 1978, na atual República do Cazaquistão (ex União Soviética), aconteceu sob a chancela da OMS a Conferência Internacional sobre Cuidados Primários de Saúde, e nesse evento foi elaborada a Declaração de Alma-Ata, que reconheceu a colaboração dos praticantes das medicinas tradicionais nos cuidados primários na saúde, recomendo-os aos estados-membro da $\mathrm{OMS}^{8}$.

Passou a haver, portanto, a recomendação para que os países membros da OMS adicionassem os métodos de medicina tradicional nas suas políticas públicas de saúde. A partir de então, houve o entendimento que as práticas integrativas e complementarem de saúde devem incluir não somente as medicinas orientais e/ou as medicinas tradicionais regionais e populares, mas também a medicina homeopática.

A Organização Mundial de Saúde (OMS), em conjunto com outros organismos internacionais como a organização Pan-Americana de Saúde (OPAS) passou a recomendar que os Estados nacionais considerassem os recursos e os praticantes das medicinas tradicionais na organização da atenção primária à saúde, tendo em vista a melhoria das condições de saúde da população ${ }^{9}$.

A partir de então, as medicinas tradicionais foram incluídas em sistemas de saúde de forma desconexa, sem diretrizes e registros das atividades. Esta é a razão pela qual, em 2002 foi lançado o documento "Estratégia da OMS sobre Medicina Tradicional 2002-2005"10", que reafirma a recomendação da OMS para que os países membros formulem seus planos, programas, projetos e políticas de saúde observando as práticas integrativas e complementares oriundas das suas populações autóctones. O documento mencionado possibilitou uma gestão mais ampliada dos programas de Medicinas Tradicionais, favorecendo uma análise dos benefícios e desafios da implementação destas práticas.

Como seguimento dessa política, no ano de 2014 reforçou-se a recomendação da OMS através de um novo documento, "A Estratégia da OMS sobre Medicina Tradicional" para o período compreendido entre 2014-2023. Nesse novo, e ainda vigente documento, a entidade revalida o seu interesse pelo desenvolvimento de políticas de saúde pública formuladas através de práticas medicinais tradicionais, tendo como foco:

7 Saad e Lima, 2010, p. $210<$ http://apps.einstein.br/revista/arquivos/PDF/1883-EC_V8_N4_p210-11.pdf>.

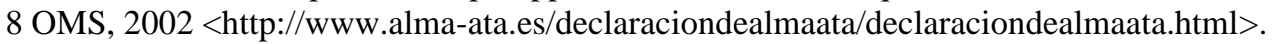

$9 \quad$ Ferreira, $2013 \quad<$ http://www.scielo.br/scielo.php?script=sci_arttext\&pid=S0104$59702013000100011 \& \operatorname{lng}=$ en\&nrm=iso $>$.

10 OMS, $2002<$ https://www.paho.org/bra/index.php?option=com_docman\&view=document\&slug=estrategia-omssobre-medicina-tradicional-2002-2005-6\&layout=default\&alias=796-estrategia-oms-sobre-medicina-tradicional2002-2005-6\&category_slug=vigilancia-sanitaria-959\&Itemid=965>. 
A ajuda às autoridades sanitárias a encontrar soluções que propiciam uma visão mais ampla a respeito da melhora da saúde e a autonomia dos pacientes. A estratégia tem dois objetivos principais: prestar apoio aos Estados Membros para que aproveitem a possível contribuição da medicina tradicional e complementar (MCT) à saúde, bem-estar e a atenção centrada nas pessoas, e promover a utilização segura e eficaz da MTC mediante a regulamentação de produtos, práticas e professionais ${ }^{11}$.

Como se observa, há, portanto, um choque de paradigmas no que tange ao cuidado à saúde básica. Na medicina moderna o doente é o paciente e o médico é o agente externo que através da tecnologia e medicamentos sintéticos irá curar a enfermidade. No outro lado desse paradigma está a medicina tradicional, que se baseia na precaução e prevenção de doenças e a través da qual o próprio doente pode realizar um autocuidado prévio a partir de um saber tradicional e popular acumulado culturalmente, tendo a eficácia dessa forma de tratamento médico comprovada através do tempo. Como ocorre com a medicina tecnologizada e medicamentosa que cada vez mais é estandarte essa forma de cuidado, o uso de fitoterápicos e plantas medicinais torna-se relevante quer seja nas pesquisas científicas quer na institucionalização dessas práticas nas políticas de saúde, complementando a medicina moderna.

Como exemplo de pesquisas científicas que comprovem a eficácia das plantas citamos o documento "Formulário de Fitoterápicos Farmacopeia Brasileira" 12 produzido pelo Ministério da Saúde do Brasil. Nesse documento há recomendações de uso e diversas outras informações sobre uma série de medicamentos de base fitoterápica que foram analisados pela Fundação Oswaldo Cruz e por órgãos de pesquisa renomados no Brasil vinculados à reconhecidas universidades federais, estaduais, como a dos estados do Amapá, Paraná e São Paulo, e das cidades de Campina Grande/PB, Ribeirão Preto/SP e Santa Maria/RS.

A medicina moderna e a tecnologia aplicada ao seu uso são de extrema importância para o tratamento de diversas enfermidades, mas, em alguns casos suas bases e práticas não contemplam a saúde e a prevenção de doenças, porque o seu foco principal é o tratamento de doenças já instaladas. Por isso a importância de complementar os serviços públicos com medicinas que abrangem outras lógicas de cuidado.

No Brasil, o uso de plantas medicinais começou com as práticas indígenas que foi influenciada pela miscigenação com as culturas africana e portuguesa o que enriqueceu ainda mais as formas de tratamento através desse método. Figueredo e colaboradores explicam que o Brasil possui uma rica história de uso das plantas medicinais e fitoterápicos no tratamento de doenças e que a perpetuação desse conhecimento se deu sobremaneira através da história oral ${ }^{13}$. A partir de meados do século XX houve o declínio dessa forma de tratamento de doenças devido a intensificação do uso de medicamentos industrializados, que em um primeiro momento eram

11 OMS, 2014 <https://www.who.int/medicines/publications/traditional/trm_strategy14_23/en/>. 12 ANVISA, 2011

<http://portal.anvisa.gov.br/documents/33832/259456/Formulario_de_Fitoterapicos_da_Farmacopeia_Brasileira.pdf/ c76283eb-29f6-4b15-8755-2073e5b4c5bf>.

13 Figueredo et. al., $2014 \quad<$ http://www.scielo.br/scielo.php?script=sci_arttext\&pid=S0103-

$73312014000200381 \& \operatorname{lng}=\mathrm{en} \& \mathrm{nrm}=\mathrm{iso}>$. 
Estudos e pesquisas sobre o uso de plantas medicinais e fitoterápicos...

importados e que posteriormente passaram a ser produzidos com pagamento de royalties de patentes para os laboratórios internacionais.

Nas políticas de saúde no Brasil, as primeiras iniciativas de inclusão das plantas medicinais e fitoterápicos no Sistema Único de Saúde, assim como o interesse pelas pesquisas envolvendo a eficácia e toxidade das plantas são anteriores à criação da Política Nacional de Plantas Medicinais e Fitoterápicos, instituída em 2006. Autores como Marcelo Saad e Paulo de Tarso Lima ${ }^{14}$ e Jairnilson Silva Paim ${ }^{15}$ afirmam que o Brasil está na vanguarda desse tema desde o movimento "Reforma Sanitária Brasileira", um movimento social urbano e democrático surgido ainda na década de 1970 e que tinha como uma das suas propostas a democratização da saúde nas periferias das grandes cidades brasileiras através do resgate às origens a práticas da medicina tradicional.

Esse movimento, segundo os autores acima citados, lançou as bases da área de saúde na Constituição Federal de 1988, sistematizando princípios e proposições que posteriormente seriam as bases à formulação do atual sistema de saúde do país. Conforme o artigo $6^{\circ}$ da Constituição Federal: "A saúde é direito de todos e dever do Estado, garantido mediante políticas sociais e econômicas que visem à redução do risco de doença e de outros agravos e ao acesso às ações e serviços para a promoção, proteção e recuperação"16.

No ano de 2008 o Governo Federal institui o "Programa Nacional de Plantas Medicinais e Fitoterápicos" que traz como objetivos: inserir plantas medicinais e fitoterápicos nos serviços oferecidos pelo SUS com segurança e eficácia; promover a inclusão da agricultura familiar nas cadeias e nos arranjos produtivos das plantas medicinais, insumos e fitoterápicos; estabelecer mecanismos de incentivo ao desenvolvimento sustentável das cadeias produtivas de plantas medicinais e fitoterápicos, com vistas ao fortalecimento da indústria farmacêutica nacional e incremento das exportações de fitoterápicos e insumos relacionados ${ }^{17}$.

A partir desse programa esperou-se que houvesse o maior uso dessas plantas no cuidado à saúde principalmente pela população que recorre ao SUS. Também, que ocorresse o desenvolvimento da indústria farmacêutica nacional no sentido de produzir remédios com elementos da flora e fauna brasileira, visto que Brasil detém entre $15 \%$ e $20 \%$ da biodiversidade do planeta. Nesse sentido, a institucionalização dos saberes, conhecimentos e práticas tradicionais e populares através do uso de plantas medicinais e fitoterápicos são fundamentais para normalizar a relação homem-meio ambiente e, de certa forma, reduzir a intensidade do impacto ambiental causado pela ação do homem no meio natural.

Assim, foram criadas políticas públicas incluindo os conhecimentos, saberes e as práticas tradicionais. Para Cleber Alho, a biodiversidade é parte importante do sistema natural dinâmico

14 Saad e Lima, 2010 <http://apps.einstein.br/revista/arquivos/PDF/1883-EC_V8_N4_p210-11.pdf〉.

15 Paim, $2008<$ http://books.scielo.org/id/4ndgv/pdf/paim-9788575413593.pdf >.

16 Brasil, 2019 <http://www.planalto.gov.br/ccivil_03/Constituicao/Constituicao.htm>.

17Ministério da Saúde, 2008

〈http://bvsms.saude.gov.br/bvs/publicacoes/programa_nacional_plantas_medicinais_fitoterapicos.pdf> 
em estrutura e função. $\mathrm{O}$ entendimento do ecossistema implica um enfoque interdisciplinar, com ênfase holística, já que é um sistema natural complexo ${ }^{18}$.

Conscientes da dualidade dos interesses do Estado e do próprio Sistema Público de Saúde brasileiro (SUS), já que este apresenta caráter dual no provimento público-privado, na Constituição Federal consta que as ações e serviços de saúde podem ser executados também por pessoa física ou jurídica de direito privado ${ }^{19}$. Tal dualidade permite que de um lado a saúde seja vista como um bem social e coletivo e por outro como um bem econômico. A política de plantas medicinais acompanha esta dualidade visando, além da busca por cuidados integrativos, a valorização do conhecimento tradicional, da biodiversidade, do autocuidado e do fomento a indústria de medicamentos fitoterápicos e dos interesses do mercado.

O desafio maior da institucionalização desta prática se encontra na forma como esta política pública pode vir a favorecer em primeiro plano a indústria farmacêutica e ao mercado de fitoterápicos das multinacionais, deixando a valorização do saber popular em segundo plano. Alguns estudos recentes sobre o uso das plantas medicinais em unidades de saúde do SUS, apontam para o aumento do uso de fitoterápicos industrializados em detrimento ao uso das plantas in natura, impulsionando assim um monopólio na fabricação dos fitoterápicos por empresas estrangeiras e multinacionais. Maria do Carmo Gullaci Guimarães Caccia-Bava e colaboradores constataram que os medicamentos fitoterápicos industrializados têm sido mais utilizados que as plantas medicinais e/ou medicamentos fitoterápicos manipulados ${ }^{20}$. Lia Hasenclever e colaboradores mostraram que entre os anos de 2009 a 2015, as 25 empresas produtoras nacionais de fitoterápicos foram compradas por 10 empresas estrangeiras, o que demonstra a crescente atratividade deste setor por parte das multinacionais ${ }^{21}$.

A próxima secção apresenta páginas eletrônicas que selecionamos e que abordam o uso de plantas medicinais e fitoterápicos. Salientamos que há outras e distintas fontes disponíveis sobre uso de plantas medicinais e fitoterápicos produzidas no Brasil e em diversos outros países, que podem e devem ser consultadas. No entanto, o que analisamos a seguir é fruto de nossas buscas no meio virtual e se constituem em uma opção para quem se interessa pela coleta de dados, informações, estatísticas, notícias e análises sobre o tema investigado.

\section{Páginas eletrônicas sobre uso de plantas medicinais e fitoterápicos}

A proposta dessa segunda secção é auxiliar o leitor na busca de dados e informações sobre as plantas medicinais e fitoterápicos. As referências indicadas abrangem as esferas da administração pública brasileira, o meio acadêmico, o setor privado, as entidades não governamentais, os movimentos sociais e demais fontes de divulgação mista. Ainda, apresentaremos portais internacionais hospedados em países que se destacam no uso de tratamento a doenças através dessa forma de medicina tradicional, baseada em práticas e saberes populares.

18 Alho, $2012<$ <ttp://www.scielo.br/pdf/ea/v26n74/a11v26n74.pdf>.

19 Brasil, 2019 <http://www.planalto.gov.br/ccivil_03/Constituicao/Constituicao.htm>.

20 Caccia-Bava et. al., 2017 <https://dx.doi.org/10.1590/1413-81232017225.16722015>.

21 Hasenclever et. al., 2016 <https://www.scielosp.org/article/csc/2017.v22n8/2559-2569/en/>. 
Estudos e pesquisas sobre o uso de plantas medicinais e fitoterápicos...

\section{Documentos do Governo Federal brasileiro}

Através da portaria n ${ }^{\circ}$ 971, de 3 de maio de 2006, o Ministério da Saúde publicou a "Política Nacional de Práticas Integrativas e Complementares da Saúde" ${ }^{22}$. Essa política inseriu na Rede de Atenção à Saúde do SUS, nos seus mais de nove mil postos de atendimento, abordagens de cuidado integral à população por meio de sistemas complexos e outras práticas que envolvem recursos terapêuticos diversos. Conforme o ministério, o SUS oferece atualmente 29 recursos terapêuticos entre suas práticas integrativas, o que demonstra a variedade de possibilidades de tratamento através de práticas que englobam o uso de plantas medicinais e fitoterápicos. São elas: o termalismo Social/Crenoterapia, práticas corporais, práticas mentais, orientação alimentar e fitoterapia tradicional chinesa. Também, ayurveda, homeopatia, Medicina Tradicional Chinesa, medicina antroposófica, plantas medicinais/fitoterapia, entre outras terapias.

Com relação às Webs que tratam do uso de plantas medicinais e fitoterápicos no Brasil, iniciaremos com a Política Nacional de Plantas Medicinais e Fitoterápicos, decreto $\mathrm{n}^{\circ} 5.813$, de 22 de junho de $2006^{23}$. Produzido pelo Ministério da Saúde, o documento é síntese e base da política pública brasileira para o uso das plantas e fitoterápicos no tratamento e atenção à saúde básica disponível no sistema público de saúde do país. Nas suas 60 páginas, a política nacional dispõe seus objetivos bem como apresenta as estratégias de monitoramento, avaliação e responsabilidades institucionais. Nas suas diretrizes e linhas prioritárias, a política nacional estabelece:

O desenvolvimento de ações pelos diversos parceiros em torno de objetivos comuns voltados à garantia do acesso seguro e uso racional de plantas medicinais e fitoterápicos em nosso país, ao desenvolvimento de tecnologias e inovações, assim como ao fortalecimento das cadeias e dos arranjos produtivos, ao uso sustentável da biodiversidade brasileira e ao desenvolvimento do Complexo Produtivo da Saúde (p. 10).

Outro documento fundamental que marca a institucionalização do uso de plantas medicinais pelo sistema público de saúde no Brasil é o "Programa Nacional de Plantas Medicinais e Fitoterápicos", criado através de portaria interministerial no 2960 de 9 de dezembro de $2008^{24}$. Também formulado pelo Ministério da Saúde, em parceria com outros ministérios, o extenso documento traz nas suas 140 páginas os seus princípios orientadores, objetivos, diretrizes e sub diretrizes, ações, gestores envolvidos, prazos e recursos. Ainda, aborda formas de monitoramento, avaliação e grupos de trabalho interministerial responsável pelo desenvolvimento do programa nos 27 estados e 5570 municípios brasileiros.

O quarto documento elaborado pelo Ministério da Saúde e que recomendamos a consulta para quem quer conhecer o processo de institucionalização do uso de plantas medicinais e fitoterápicos no Brasil é o intitulado "A fitoterapia no SUS e o Programa de Pesquisa de Plantas Medicinais da Central de Medicamentos ${ }^{25}$ ". Esse documento é relevante já que apresenta a historicidade dos programas oficiais de apoio à pesquisa científica e uso de plantas medicinais no

22 Ministério da Saúde, 2006c <http://bvsms.saude.gov.br/bvs/saudelegis/gm/2006/prt0971_03_05_2006.html>.

23 Ministério da Saúde, 2006 <http://bvsms.saude.gov.br/bvs/publicacoes/politica_nacional_fitoterapicos.pdf>.

24 Ministério da Saúde, 2009.

〈http://bvsms.saude.gov.br/bvs/publicacoes/programa_nacional_plantas_medicinais_fitoterapicos.pdf>.

25 Ministério da Saúde, 2006b <http://bvsms.saude.gov.br/bvs/publicacoes/fitoterapia_no_sus.pdf >. 
país. Aborda a atuação da Central de Medicamentos (CEME), programa do Governo Federal criado em 1971 e que tinha como missão o fornecimento de medicamentos à população de baixa renda, o que o caracterizava por manter uma política centralizada de aquisição e distribuição de medicamentos. A CEME foi extinta e no seu lugar foi criado o "Programa de Pesquisas de Plantas Medicinais" (PPPM), no ano de 1997. O PPPM, que deu origem ao "Programa Nacional de Plantas Medicinais e Fitoterápicos" atual, tinha como objetivo principal a promoção, a pesquisa científica, o incentivo e difusão do uso de plantas medicinais para a população.

Ressaltamos a praticidade e facilidade de compreensão dos conteúdos das páginas eletrônicas do Ministério da Saúde do Brasil apresentadas acima, de forma que tanto um especialista da área, quanto um cidadão leigo no tema, podem compreender a intenção do Governo Federal no que tange à política de uso de plantas medicinais e fitoterápicos no Sistema Único de Saúde como prática complementar no cuidado e atenção à saúde básica.

\section{Portais de Pesquisa de Instituições Públicas Brasileiras}

O primeiro portal de divulgação de pesquisas científicas sobre plantas medicinais e fitoterápicos que recomendamos a consulta é o Flora do Brasil $2020^{26}$. Signatário da Estratégia Global para a Conservação de Plantas, no ano de 2010, o Brasil conseguiu cumprir a meta 1 do referido documento, publicando o "Catálogo de Plantas e Fungos do Brasil" e, posteriormente, a primeira versão da Lista de Espécies da Flora do Brasil. Atualmente, o portal "Flora do Brasil 2020" é um poderoso motor de busca acerca das plantas medicinais justamente por conter informações sobre indicações de uso, áreas de distribuição geográfica das plantas, bem como os ecossistemas de ocorrência de todas as espécies catalogadas no país

Apesar de não descrever seus usos medicinais, Flora do Brasil 2020 é um portal de extrema relevância para o campo de pesquisa das plantas medicinais devido as informações contidas na sua página eletrônica. Como informação técnica inicial, o portal apresenta que são reconhecidas 46.673 espécies na flora brasileira, sendo distribuídas 4.756 em forma de Algas, 33.239 de Angiospermas, 1.569 de Briófitas, 5.719 de Fungos, 29 Gimnospermas e 1.361 de Samambaias e Licófitas. A limitação que o portal oferece é que somente espécies nativas podem ser consultadas, e, portanto, espécies que foram introduzidas pelos colonizadores e de uso em larga escala, caso do alho, não aparecem no referido portal.

A Agência Nacional de Vigilância Sanitária (ANVISA) é uma agência reguladora, sob a forma de autarquia vinculada ao Ministério da Saúde do Brasil. A agência exerce o controle sanitário sobre todos os produtos e serviços (nacionais e importados) submetidos à vigilância sanitária, tais como medicamentos, alimentos, cosméticos, saneantes, derivados do tabaco, produtos médicos, sangue, hemoderivados e serviços de saúde. Dentre suas atribuições, a Agência deve promover a revisão e atualização periódica da Farmacopeia Brasileira, conforme disposto no inciso XIX do artigo $7^{\circ}$ da Lei 9.782 de 26 de janeiro 1999: "Esta competência compreende ações de regulamentação sanitária e indução ao desenvolvimento científico e tecnológico nacional que se concretizam por

26 Flora do Brasil 2020, 2019

<http://floradobrasil.jbrj.gov.br/reflora/listaBrasil/PrincipalUC/PrincipalUC.do;jsessionid=B51C27D9A846B5280D C6A8DE9FBF0637\#CondicaoTaxonCP>. 
Estudos e pesquisas sobre o uso de plantas medicinais e fitoterápicos...

meio da revisão e incorporação de novos requisitos de qualidade nos compêndios e produtos da Farmacopeia Brasileira”.

No ano de 2011, a ANVISA lançou o primeiro "Formulário de fitoterápicos, farmacopeia brasileira" 27 . Esse formulário contém receitas de pomadas, tinturas, xaropes e chás, elaborado pela ANVISA, para uso popular, além de incentivar a preparação de remédios caseiros produzidos com plantas medicinais de fácil acesso à população. As recomendações desse formulário se inserem no que se denomina autocuidado, ou seja, que o próprio doente possa, de maneira inicial, tentar cuidar da sua saúde através do uso de plantas medicinais e tratamento com medicamentos fitoterápicos.

No ano de 2015, a Prefeitura da cidade do Rio de Janeiro/RJ lançou o "Guia prático de plantas medicinais". Esse guia reúne informações sobre armazenamento, reconhecimento de espécies e formas de uso de dezenove plantas. Foi elaborado por especialistas com o intuito de demonstrar o uso seguro de espécies populares. Dentre as espécies mais difundidas, destacam-se: Guaco, da espécie Mikania Laevegata, utilizado para afecções respiratórias; Aroeira, da espécie Shinus terebentthifolius, utilizado para cistite e infecções vaginais; e a espinheira santa da espécie Maytenus Ilicifolia, utilizado para afecções gástricas ${ }^{28}$.

\section{Revistas de Divulgação Científica}

No que tange às revistas científicas que divulgam especificamente pesquisas voltadas à área de plantas medicinais e fitoterápicos, a primeira que recomendamos a consulta ao seu portal eletrônico é a Revista Brasileira de Plantas Medicinais ${ }^{29}$. Indexada na plataforma Scielo, a revista é mantida pela Sociedade Brasileira de Plantas Medicinais e possui elevado reconhecimento acadêmico chancelado pela Coordenação de Aperfeiçoamento de Pessoal de Nível Superior (CAPES) e Qualis Periódicos ${ }^{30}$.

A Fundação Oswaldo Cruz (FIOCRUZ) é uma instituição pública do Governo Federal de pesquisa e desenvolvimento em ciências biológicas, que atualmente é considerada uma das principais instituições de ensino e pesquisa sobre saúde pública no mundo. Na FIOCRUZ se encontra o Laboratório Farmacêutico Federal Farmanguinhos e o seu Núcleo de Gestão em Biodiversidade e Saúde e a publicação da Revista Fitos. Esta revista é um periódico de caráter interdisciplinar, em acesso aberto, de difusão gratuita e com periodicidade trimestral. Publica artigos científicos originais sobre Pesquisa, Desenvolvimento e Inovação em medicamentos da

\footnotetext{
27 ANVISA, 2011

<http://portal.anvisa.gov.br/documents/33832/259456/Formulario_de_Fitoterapicos_da_Farmacopeia_Brasileira.pdf/ c76283eb-29f6-4b15-8755-2073e5b4c5bf>.

28 Prefeitura do Rio de Janeiro, 2015 <www.rio.rj.gov.br/documents/73801/14163dd4-f62d-4f52-8b08$81 \mathrm{ff} 3 \mathrm{f} 625212>$.

29 Revista Brasileira de Plantas Medicinais, $2019<\mathrm{http}: / / w w w . s c i e l o . b r / s c i e l o . p h p ? s c r i p t=s c i \_s e r i a l \& p i d=1516-$ $0572 \& \operatorname{lng}=$ en\&nrm=isso $>$.

30 Plataforma Sucupira, 2019

<https://sucupira.capes.gov.br/sucupira/public/consultas/coleta/veiculoPublicacaoQualis/listaConsultaGeralPeriodico s.jsf>.
} 
diversidade vegetal. Em uma consulta ao termo plantas medicinais encontramos ao menos 162 artigos; já para o termo fitoterápicos encontramos outros $41 \operatorname{artigos}^{31}$.

Os artigos que são publicados na Revista Fitos tratam da temática plantas medicinais e fitoterápicos através de abordagens tais como: Agroecologia, Botânica, Ciências Farmacêuticas, Educação, Etnociências, Engenharia de Medicamentos, Produtos Naturais, Farmacologia, Políticas Públicas, Política e Planejamento Governamental e Saúde Pública e Toxicologia. Em sua última edição em 2019 a revista disponibilizou artigos como: "Fitoterápicos na atenção primária da saúde: desafios e perspectivas na atuação médica na saúde" e "Levantamento etnobotânico de plantas medicinais em comunidades rurais no município de Lagoa Grande, Pernambuco, Brasil". Esses artigos apresentam resultados comprovados em laboratórios sobre a eficácia do uso das plantas e dos medicamentos fitoterápicos no tratamento de doenças

A Revista Revise. Revista Integrativa nas Inovações Tecnológicas das Ciências da Saúde, da Universidade Federal do Recôncavo da Bahia, possui como foco as publicações na área das Práticas Integrativas e Complementares da Saúde além de outros temas importantes para o campo das inovações sociais educacionais em saúde. Ela visa promover uma visão dialógica e integrativa entre os diferentes campos do conhecimento que transitam entre a educação e a saúde, porém ainda se encontra em fase de indexação ${ }^{32}$.

A revista Planta Médica é mais uma opção de pesquisa acadêmica sobre o tema por ser considerada uma das principais revistas internacionais no campo dos produtos naturais incluindo organismos marinhos, fungos, bem como microrganismos - e plantas medicinais. A revista publica 18 edições por ano nas seguintes áreas de plantas medicinais e pesquisa de produtos naturais são abordadas: atividades biológicas, farmacológicas e química de produtos naturais, estudos analíticos, investigações farmacocinéticas e Sistemas de formulação e entrega de produtos naturais. Uma especificidade dessa revista em relação as demais é o fato de ser paga a disponibilização de seus números completos e $\operatorname{artigos}^{33}$.

\section{Organizações Não Governamentais e Entidades do Terceiro Setor}

A Sociedade Brasileira de Plantas Medicinais (SBPM) ${ }^{34}$ foi criada no ano de 1988 e possui como missão agregar profissionais de diferentes áreas do conhecimento que desenvolvam as suas atividades na área de plantas medicinais. Além da Revista Brasileira de Plantas Medicinais, a SBPM promove a cada dois anos o Simpósio de Plantas Medicinais do Brasil, cuja sua XXVI edição será realizada no ano de 2020. O último simpósio aconteceu em 2018 e trouxe como tema principal a necessidade de pesquisa, manipulação, produção, aproveitamento adequado e conservação das plantas medicinais utilizadas no território nacional.

31 Revista Fitos, 2019 <http://www.revistafitos.far.fiocruz.br/index.php/revista-fitos>.

32 Revista Revise, $2019<$ http://www.ufrb.edu.br/saed/revista-revise>.

33 Revista Planta Médica, 2019 <https://www.thieme.in/planta-medica>.

34 SBPM, 2019 <http://www.sbpmed.org.br/index.php>. 
Estudos e pesquisas sobre o uso de plantas medicinais e fitoterápicos...

Análogo a SBPM, a Associação Brasileira de Fitoterapia (ABFIT) ${ }^{35}$ é uma associação do terceiro setor também criada no ano de 1988 e que congrega os profissionais da fitoterapia. Conforme seus objetivos, a ABFIT se dedica ao desenvolvimento tecnológico e científico de processos e procedimentos relacionados com o aproveitamento das plantas medicinais, com a conservação da biodiversidade brasileira bem como a preservação das culturas tradicionais dos povos nativos no país. Com um caráter mais técnico, a ABFIT se destaca pelo oferecimento de diversos cursos de formação, como por exemplo, o curso de especialização em fitoterapia, bem como na disponibilização de informações sobre o mercado laboral para profissionais de fitoterapia no país.

A ABFIT ainda mantém um portal eletrônico chamado "Tudo sobre plantas"36, com acesso gratuito. Consta de um poderoso banco de dados de informações sobre plantas medicinais já identificadas, catalogadas e com especificações de uso a partir dos testes químicos e biológicos já comprovados por diversas outras instituições e pelos próprios membros da associação.

A Comissão Pró-Índio de São Paulo é uma Organização Não Governamental que monitora a questão da posse de terra dos povos indígenas do Brasil. No ano de 2014 a ONG publicou o documento intitulado "Folhas e raízes: resgatando a medicina tradicional Tupi-Guaran"37. A cartilha foi elaborada por professores da aldeia Piaçaguera Ywy Pyaú, Luan Elísio Apyká e Dhevan Pacheco, com o apoio de seus alunos e das pessoas mais idosas da aldeia, os txeramoi e txedjaryi, que são os que conhecem o uso medicinal das plantas, em especial o pajé e Amâncio Samuel dos Santos Rókenedju. A proposta dessa cartilha é o de compartilhar o que os TupiGuaranis chamam de medicina do mato, ou seja, o aprendizado no uso das plantas que vão adquirindo a partir do contato direto com a natureza. A cartilha traz ainda dicas de como utilizar ervas, folhas e raízes encontradas nas cinco macrorregiões brasileiras.

A Organização Não Governamental Entre Folhas ${ }^{38}$, com sede na Universidade Federal de Viçosa, estado de Minas Gerais, foi fundada em 2010 e possui como objetivos a valorização da sabedoria popular, o desenvolvimento de pesquisas sobre as diversas práticas integrativas, além da promoção de cursos e oficinas com o intuito de oferecer conhecimento sobre tais práticas da saúde. A ONG possui um acervo relevante de trabalhos desenvolvidos pelos seus integrantes e documentos acadêmicos, oficiais e demais publicações sobre o uso de plantas medicinais e fitoterápicos.

Outro portal eletrônico que vale a pena consultar é o da Associação Brasileira das Empresas do Setor Fitoterápico, Suplemento Alimentar e de Promoção da Saúde (ABIFISA). Esta associação foi fundada em 2000 e congrega empresas do setor fitoterápico, atuando como entidade representativa do setor. A ABIFISA faz parte da Câmara Setorial de Medicamentos da Câmara dos Deputados e é representante do Ministério do Desenvolvimento, Indústria e Comércio no Comitê Nacional de Plantas Medicinais e Fitoterápicos. Também faz parte da Rede Fito Amazônia, da Rede Fito Mata Atlântica e de outras Redes dos Biomas, do Fórum de

35 ABFIT, 2019 <https://www.abfit.org.br/sobre-a-abfit>.

36 Tudo sobre planta, $2019<$ www.tudosobreplanta.com.br $>$.

37 Comissão Pró-Índio, 2014 <https://nossofuturoroubado.com.br/cartilha-folhas-e-raizes-medicina-indigena-tupiguarani/>.

38 Entre folhas, $2019<$ http://entrefolhaspi.eco.br/>. 
Competitividade da Cadeia Produtiva Farmacêutica no Brasil, do Fórum de Competitividade da Biotecnologia, da Comissão de Revisão da Farmacopeia Brasileira, do Comitê Consultivo do Programa Regional de Apoio à Rede de Desenvolvimento de Plantas Medicinais, do Programa de Plantas Medicinais do MERCOSUL (PLAMSUR) e da Rede de Agentes em Política Industrial (RENAPI). Participa ativamente de diversos grupos de trabalho na área de fitoterápicos, alimentos e de produtos para a promoção da saúde, na ANVISA e em outras entidades ${ }^{39}$ ".

Os membros da ABIFISA contribuíram com a coordenação na edição de livros tais como Monografias de Plantas Medicinais Brasileiras ${ }^{40}$ e Coletânea Científica de Plantas de Uso Medicinal $^{41}$. Estes livros fazem parte da lista de referências bibliográficas para avaliação de segurança e eficácia de medicamentos fitoterápicos da Instrução Normativa no 05/2010, a qual regulamenta o Sistema de Licenciamento de Atividades Poluidoras ou Degradadoras do Meio Ambiente (SILCAP).

\section{Páginas Eletrônicas Internacionais}

No ano de 1991, o Governo Federal dos Estados Unidos criou o Nacional Center for Complementary and Alternative Medicine (NCCCAM) com o objetivo de pesquisar, sistematizar e avaliar as práticas alternativas em saúde ${ }^{42}$. Atualmente foram tomadas medidas para fortalecer as disposições das estruturas regulatórias para fabricação de suplementos alimentares. A comissão federal de comércio publicou diretrizes e controle da publicidade comercial para complementos dietéticos, principalmente os de origem botânica.

$\mathrm{Na}$ Europa, as formas de reconhecimento e regulação de tratamento de doenças através de plantas medicinais e uso de fitoterápicos são diversos. No ano de 1997, a Comissão de Meio Ambiente, Saúde Pública e Proteção do Consumidor do Parlamento Europeu aprovou uma proposta de resolução sobre o uso de medicinas alternativas. Em 2012 foi estabelecida a rede europeia de pesquisa CAMbrella que tem por objetivo avaliar as condições que sustentam o uso das Medicinas Tradicionais e Complementares e o fornecimento de seus produtos na Europa ${ }^{43}$. A rede de pesquisa CAMbrella congrega 16 grupos de pesquisa acadêmica em 12 países europeus. Conforme a OMS no seu já citado documento "A Estratégia da OMS sobre Medicina Tradicional", na União Europeia a legislação sobre as medicinas tradicionais e complementares vem desenvolvendo uma abordagem harmonizada para a regulamentação de fitoterápicos.

$\mathrm{Na}$ América Latina, além do Brasil, países como Argentina ${ }^{44}$, Bolívia ${ }^{45}$, Chile ${ }^{46}$, Colômbia ${ }^{47}$, $\mathrm{Cuba}^{48}$, México $^{49}$ e Peru ${ }^{50}$ possuem legislações e implementações diversas nos seus sistemas de

39 ABIFISA, 2019 <http://www.abifisa.org.br/quem-somos>.

40 Gilbert et. al., 2005.

41 Amaral et. al., 2005.

42 NCCCAM, $2019<$ https://nccih.nih.gov/>.

43 CAMbrella, 2019 <https://cam-europe.eu/library-cam/cambrella-research-reports>.

44 Senado de Argentina, 2016<http://www.senado.gov.ar/parlamentario/parlamentaria/383747/downloadPdf >.

45 Ministerio de Salud y Deportes, 2013

<http://www.sipi.siteal.iipe.unesco.org/sites/default/files/sipi_intervencion/safci.pdf>.

46 Ministerio de Salud de Chile, 2019 <https:/www.minsal.cl/medicinas-complementarias/>. 
Estudos e pesquisas sobre o uso de plantas medicinais e fitoterápicos...

saúde e que contemplam, como parte do sistema formal de saúde o uso de plantas medicinais e fisioterápicos. No Equador ${ }^{51}$, a inovação na sua política de medicinas tradicionais e uso de plantas e fitoterápicos no cuidado à saúde está no fato dela estar atrelada a preceitos constitucionais de Buen Vivir y el Vivir Bueno, ou seja, que o cidadão equatoriano tem o direito de viver em plenitude, em harmonia e equilíbrio com a natureza, e em comunidade.

\section{Considerações Finais}

Ao longo do texto mostramos diversos portais eletrônicos que tratam do uso de plantas medicinais e fitoterápicos no cuidado à saúde, porém evitamos apresentar centenas de páginas eletrônicas de modo a tornar impossível a navegação e coleta de informações, muitas delas desconexas, disponibilizadas no mundo virtual. Porém, a partir de estudos já realizados sobre o tema indicamos referências que tratam de aspectos históricos, institucionais e analíticos e, nesse interim, foi dado um enfoque especial a referências formuladas no Brasil.

Analisamos também a institucionalização do uso de plantas medicinais e fitoterápicos pelos países membros da OMS, com ênfase no Brasil através da Política Nacional de Práticas Integrativas e Complementares.

A institucionalização de práticas medicinais consideradas tradicionais proporciona um novo olhar por parte do Estado no que tange aos cuidados com a saúde humana, reconhecendo a integralidade da saúde, resgatando outras possibilidades de cuidado mais relacionados aos saberes e conhecimentos construídos ao longo dos séculos pelas populações autóctones. A promoção de uma política pública na área da saúde que contemple o uso de plantas medicinais e medicamentos fitoterápicos implica na necessidade de um maior cuidado à biodiversidade do país.

Consideramos como os maiores desafios para o desenvolvimento dessas práticas a descrença e o desconhecimento dos profissionais sobre o uso das plantas. Outro obstáculo é a apropriação dessa prática de conhecimento popular e tradicional pelo mercado, uma vez que a distribuição de fitoterápicos industrializados vem superando o uso das plantas in natura. $\mathrm{O}$ crescimento da fabricação e distribuição de fitoterápicos industrializados transforma o símbolo de cuidado das medicinas tradicionais e populares alinhando aos mesmos preceitos prescritivos da biomedicina. Neste contexto, a indústria de fitoterápicos passa a ser um negócio a mais da indústria farmacêutica.

\footnotetext{
47 INVIMA, $2019 \quad<$ https://www.invima.gov.co/conformacion-de-las-salas-especializadas/242-salasespecializadas/sala-especializada-de-productos-naturales/3259-documentos-tecnicos.html>. 48 Ministerio de Salud Pública, 2009 <https://www.cecmed.cu/sites/default/files/adjuntos/Reglamentacion/Res_MINSAP-321-09.pdf>.

49 COFEPRIS, 2019 <https://www.gob.mx/cofepris/prensa/cofepris-anuncia-la-liberacion-de-18-plantasmedicinales-para-su-uso-legal?idiom=es $>$.

50 Instituto Nacional de Salud, 2019 <https://web.ins.gob.pe/es/salud-intercultural/medicina-tradicional/plantasmedicinales>.

51 Ministerio de Salud Púbica, 2014 <http://www.calidadsalud.gob.ec/wp-content/uploads/2017/08/EJERCICIOMEDICINA-ALTERNATIVA-ac_00005001_2014-13-ago.pdf>
} 


\section{Recursos digitais e bibliográficos}

ABFIT. Associação Brasileira de Fitoterapia. 2019 <https://www.abfit.org.br/sobre-a-abfit>. [30 de janeiro de 2019].

ABIFISA. Associação Brasileira das Empresas do Setor Fitoterápico, Suplemento Alimentar e de Promoção da Saúde. [En línea]. 2019 <http://www.abifisa.org.br/quem-somos>. [30 de janeiro de 2019].

ALHO, Cleber. Importância da Biodiversidade para a saúde humana: uma perspectiva $\begin{array}{lllll}\text { ecológica. } & \text { Rev. } & \text { estudos } & 26 & \text { (74). }\end{array}$ <http://www.scielo.br/pdf/ea/v26n74/a11v26n74.pdf>. [30 de janeiro de 2019].

ALMA-ATA, 1978 <http://www.alma-ata.es/declaraciondealmaata/declaraciondealmaata.html>

AMARAL, A. C. F.; SIMÕES, E. V.; FERREIRA, J. L. P. Coletânea científica de plantas de uso medicinal. Rio de Janeiro: FIOCRUZ, 2005.

ANVISA. Formulário de fitoterápicos, farmacopeia brasileira. Brasília: Agência Nacional de Vigilância Sanitária, 2011 <http://portal.anvisa.gov.br/documents/33832/259456/Formulario_de_Fitoterapicos_da_Farmaco peia_Brasileira.pdf/c76283eb-29f6-4b15-8755-2073e5b4c5bf>. [30 de janeiro de 2019].

BRASIL. Constituição $\quad$ Federal. $\quad$ Brasília: 2019 <http://www.planalto.gov.br/ccivil_03/Constituicao/Constituicao.htm>. [30 de janeiro de 2019].

CACCIA-BAVA, Maria do Carmo Gullaci Guimarães; BERTONI, Bianca Waléria; PEREIRA, Ana Maria Soares; MARTINEZ, Edson Zangiacomi. Disponibilidade de medicamentos fitoterápicos e plantas medicinais nas unidades de atenção básica do Estado de São Paulo: resultados do Programa Nacional de Melhoria do Acesso e da Qualidade da Atenção Básica (PMAQ). Ciência \& Saúde Coletiva, 22(5), 1651-1659. $2017<\mathrm{https}: / / d x . d o i . o r g / 10.1590 / 1413-$ 81232017225.16722015>. [15 de novembro de 2019].

CAMBRELLA. The roadmap for European CAM Research: an Explanation of the CAM Projectand its key findin. 2019 <https://cam-europe.eu/library-cam/cambrella-research-reports>. [30 de janeiro de 2019].

CAPEL, Horacio. Una geografía para el siglo XXI. Scripta Nova, Revista Electrónica de Geografía y Ciencias Sociales. Barcelona: Universidad de Barcelona, $\mathrm{n}^{\circ}$ 19, 1998 <http://www.ub.es/geocrit/sn-19.htm>. [30 de janeiro de 2019].

CASTRO, Marta Rocha; FIGUEIREDO, Fábio Fonseca. Saberes tradicionais, biodiversidade, práticas integrativas e complementares: o uso de plantas medicinais no SUS. Hygeia, Revista Brasileira de Geografia Médica e da Saúde. Uberlândia: Universidade Federal de Uberlândia, 15 (31): 56-70, Março/2019 <http://www.seer.ufu.br/index.php/hygeia/article/view/46605/26405>. [15 de novembro de 2019]. 
Estudos e pesquisas sobre o uso de plantas medicinais e fitoterápicos...

COFEPRIS. Comisión Federal para la Protección Contra Riesgos Sanitario. Ciudad de México: $2019<$ https://www.gob.mx/cofepris/prensa/cofepris-anuncia-la-liberacion-de-18-plantasmedicinales-para-su-uso-legal?idiom=es >. [30 de janeiro de 2019].

COMISSÃO PRÓ-ÍNDIO. São Paulo: Folhas e raízes: resgatando a medicina tradicional TupiGuarani, $2014<$ https://nossofuturoroubado.com.br/cartilha-folhas-e-raizes-medicina-indigenatupi-guarani/>. [30 de janeiro de 2019].

ENTRE FOLHAS. Viçosa: 2019 <http://entrefolhaspi.eco.br/>. [30 de janeiro de 2019].

FIGUEREDO, Climério Avelino de; GURGEL, Idê Gomes Dantas; GURGEL JUNIOR, Garibaldi Dantas. A Política Nacional de Plantas Medicinais e Fitoterápicos: construção, perspectivas e desafios. Physis, 2014, vol.24, $\mathrm{n}^{\circ} 2$ p. 381-400 <http://www.scielo.br/scielo.php?script=sci_arttext\&pid=S0103-

73312014000200381\&lng=en\&nrm=iso>. [30 de janeiro de 2019].

FERREIRA, Luciane Ouriques. A emergência da medicina tradicional indígena no campo das políticas públicas. Hist. cienc. Saúde Manguinhos. Rio de Janeiro: v. 20, no 1, p. 203-219, 2013 <http://www.scielo.br/scielo.php?script=sci_arttext\&pid=S010459702013000100011\&lng=en\&nrm=iso>. [30 de janeiro de 2019].

$\begin{array}{lllll}\text { FLORA } & \text { DO } & \text { BRASIL } & 2020 . & \text { Brasília: }\end{array}$ <http://floradobrasil.jbrj.gov.br/reflora/listaBrasil/PrincipalUC/PrincipalUC.do;jsessionid=B51C 27D9A846B5280DC6A8DE9FBF0637\#CondicaoTaxonCP>. [30 de janeiro de 2019].

GILBERT, B.; FERREIRA, J. L. P.; ALVEZ, L. P. Monografias de Plantas Medicinais Brasileiras e Aclimatadas. Curitiba: ABIFIT, 2005

HASENCLEVER, Lia; PARANHOS, Julia; COSTA, Cíntia Reis; CUNHA, Gabriel; VIEIRA, D. A indústria de fitoterápicos brasileira: desafios e oportunidades. Ciência \& Saúde Coletiva. Rio de Janeiro: $\mathrm{n}^{\mathrm{o}}$ 22, p.p: 2559-2569. <https://www.scielosp.org/article/csc/2017.v22n8/25592569/en/>. [18 de novembro de 2019].

INSTITUTO NACIONAL DE SALUD. Lima: 2019 <https://web.ins.gob.pe/es/saludintercultural/medicina-tradicional/plantas-medicinales $>$. [30 de janeiro de 2019].

INVIMA. Instituto Nacional de Vigilancia de Medicamentos y Alimentos. Bogotá: 2019. $<$ https://www.invima.gov.co/conformacion-de-las-salas-especializadas/242-salasespecializadas/sala-especializada-de-productos-naturales/3259-documentos-tecnicos.html>. [30 de janeiro de 2019].

LATINDEX. México: Latindex, 2019. <http://www.latindex.unam.mx/>. [23 de janeiro de 2019]. 
MINISTÉRIO DA SAÚDE. Mais 10 práticas integrativas são inseridas na PNPIC. Brasília: $2018<$ http://dab.saude.gov.br/portaldab/noticias.php?conteudo=_\&cod=2872>. http://www.latindex.unam.mx/>. [23 de janeiro de 2019].

MINISTÉRIO DA SAÚDE. Programa Nacional de Plantas Medicinais e Fitoterápicos. Brasília: 2009

<http://bvsms.saude.gov.br/bvs/publicacoes/programa_nacional_plantas_medicinais_fitoterapico s.pdf >. [30 de janeiro de 2019].

MINISTÉRIO DA SAÚDE. Política Nacional de Plantas Medicinais e Fitoterápicos. Brasília: $2006<$ http://bvsms.saude.gov.br/bvs/publicacoes/politica_nacional_fitoterapicos.pdf>. [30 de janeiro de 2019].

MINISTÉRIO DA SAÚDE. A fitoterapia no SUS e o Programa de Pesquisa de Plantas Medicinais da Central de Medicamentos. Brasília: 2006b <http://bvsms.saude.gov.br/bvs/publicacoes/fitoterapia_no_sus.pdf >. [30 de janeiro de 2019].

MINISTÉRIO DA SAÚDE. Política Nacional de Práticas Integrativas e Complementares. Brasília: 2006c <http://bvsms.saude.gov.br/bvs/saudelegis/gm/2006/prt0971_03_05_2006.html>. [30 de janeiro de 2019].

MINISTERIO DE SALUD PÚBLICA. Resolución Ministerial $n^{o}$ 321. La Habana. 2009. <https://www.cecmed.cu/sites/default/files/adjuntos/Reglamentacion/Res_MINSAP-321-09.pdf>. [30 de janeiro de 2019].

MINISTERIO DE SALUD DE CHILE. Santiago: $2019<$ https://www.minsal.cl/medicinascomplementarias/>. [30 de janeiro de 2019].

MINISTERIO DE SALUD PÚBICA. Quito: 2014. <http://www.calidadsalud.gob.ec/wpcontent/uploads/2017/08/EJERCICIO-MEDICINA-ALTERNATIVA-ac_00005001_2014-13ago.pdf >. [30 de janeiro de 2019].

MINISTERIO DE SALUD Y DEPORTES. La política SAFCI, su estrategia de promoción de la salud $y$ prácticas alimentarias saludables. $\quad$ La Paz: 2013 <http://www.sipi.siteal.iipe.unesco.org/sites/default/files/sipi_intervencion/safci.pdf>. $\left[\begin{array}{lll}30 & \text { de }\end{array}\right.$ janeiro de 2019].

MINISTÉRIO DO MEIO AMBIENTE. Estratégia Global para a Conservação de Plantas. Brasília: 2010. <http://www.mma.gov.br/estruturas/221/_arquivos/estratgia_global_conservao_plantas_221.pdf> . [30 de janeiro de 2019].

NASCIMENTO, Marilene Cabral; BARROS, Nelson Filice; NOGUEIRA, Maria Inês; LUZ, Madel Therezinha. A categoria racionalidade médica e uma nova epistemologia em saúde. Ciência \& Saúde Coletiva. $\quad \mathrm{N}^{\mathrm{O}} \quad 18, \quad 3595-3604, \quad 2013$. <https://www.scielosp.org/article/csc/2013.v18n12/3595-3604/>. [30 de outubro de 2019]. 
Estudos e pesquisas sobre o uso de plantas medicinais e fitoterápicos...

NCCCAM. Nacional Center for Complementary and Alternative Medicine. Washington: 2019 $<$ https://nccih.nih.gov/>. [30 de janeiro de 2019].

OMS. A Estratégia da OMS sobre Medicina Tradicional. Organização Mundial da Saúde, 2014 <https://www.who.int/medicines/publications/traditional/trm_strategy14_23/en/>. [30 de janeiro de 2019].

PAIM, Jairnilson Silva. Reforma Sanitária Brasileira: contribuição para a compreensão $e$ crítica. FIOCRUZ, 2008 <http://books.scielo.org/id/4ndgv/pdf/paim-9788575413593.pdf>. [30 de janeiro de 2019].

PARLAMENTO EUROPEU. Sobre o estatuto das medicinas não convencionais. 2019 <http://www.europarl.europa.eu/sides/getDoc.do?pubRef=-//EP//NONSGML+REPORT+A41997-0075+0+DOC+PDF+V0//PT>. [30 de janeiro de 2019].

PLATAFORMA

SUCUPIRA.

<https://sucupira.capes.gov.br/sucupira/public/consultas/coleta/veiculoPublicacaoQualis/listaCon sultaGeralPeriodicos.jsf>. [30 de janeiro de 2019].

PREFEITURA DO RIO DE JANEIRO. Guia prático de plantas medicinais. Rio de Janeiro: 2015 <www.rio.rj.gov.br/documents/73801/14163dd4-f62d-4f52-8b08-81ff3f625212>. [30 de janeiro de 2019].

$\begin{array}{lllll}\text { REVISTA } & \text { BRASILEIRA } & \text { DE } & \text { PLANTAS } & \text { MEDICINAIS. }\end{array}$ $<$ http://www.scielo.br/scielo.php?script=sci_serial\&pid=1516-0572\&lng=en\&nrm=isso $>$. [30 de janeiro de 2019].

REVISTA FITOS. Brasil: 2019 <http://www.revistafitos.far.fiocruz.br/index.php/revista-fitos>. [30 de janeiro de 2019].

SAAD, Marcelo; LIMA, Paulo de Tarso. Acesso às terapias complementares cresce no SUS. Einstein: Educ. Contin. Saúde. 2010 <http://apps.einstein.br/revista/arquivos/PDF/1883EC_V8_N4_p210-11.pdf>. [30 de janeiro de 2019].

SBPM. Sociedade Brasileira de Plantas Medicinais. Brasil: 2019 <http://www.sbpmed.org.br/index.php>. [30 de janeiro de 2019].

SENADO DE ARGENTINA. Proyecto de Ley S-4579/16. Buenos Aires: 2016 <http://www.senado.gov.ar/parlamentario/parlamentaria/383747/downloadPdf>. [30 de janeiro de 2019].

TUDO SOBRE PLANTAS. Rio de Janeiro: 2019 <https://www.tudosobreplantas.com.br/asp/plantas/ficha.asp?id_planta=15632>. [30 de outubro de 2019]. 
(c) Copyright: Marta Rocha de Castro, Fabio Fonseca Figueiredo, 2020.

(C) Copyright: Ar@cne, 2020.

Ficha bibliográfica:

CASTRO, Marta Rocha de; FIGUEIREDO, Fábio Fonseca. Estudos e pesquisas sobre o uso de plantas medicinais e fitoterápicos no Brasil: caminhos e desafios.Ar@cne. Revista Electrónica de Recursos de Internet sobre Geografía y Ciencias Sociales. [En línea]. Barcelona: Universidad de Barcelona, nº 240, 1 de marzo de 2020. <http://www.ub.edu/geocrit/aracne/aracne-240.pdf>. ISSN: 1578-0007.

Menú principal de Geo Crítica

Índice de Ar@cne 